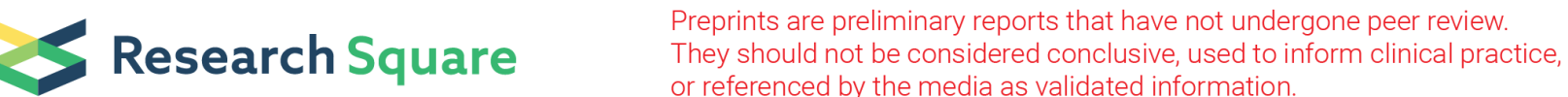

\section{The intermittent fasting-dependent gut microbial metabolite indole-3 propionate promotes nerve regeneration and recovery after injury}

Simone Di Giovanni ( $\nabla$ s.di-giovanni@imperial.ac.uk)

Imperial College London https://orcid.org/0000-0003-3154-5399

elisabeth serger

Imperial College London

Jessica Chadwick

Imperial College London

Lucia Luengo

Imperial College London

Guiping Kong

Imperial College London

Luming Zhou

Imperial College London

\section{Greg Crawford}

Imperial College London

\section{Matt Danzi}

Dr. John T. Macdonald Foundation Department of Human Genetics and John P. Hussman Institute for Human Genomics, University of Miami Miller School of Medicine

Antonis Myridakis

Imperial College London https://orcid.org/0000-0003-1690-6651

\section{Alexander Brandis}

WEIZMANN INSTITUTE

Adesola Bello

Imperial College London

Francesco De Virgiliis

Imperial College London

\section{Marc-Emmanuel Dumas}

Imperial College London https://orcid.org/0000-0001-9523-7024

Jessica Strid

Imperial College London https://orcid.org/0000-0003-3690-2201

\section{Dylan Dodd}

Stanford University https://orcid.org/0000-0001-6210-6239 


\section{Biological Sciences - Article}

Keywords: intermittent fasting, gut microbiome, nerve regeneration, injury

Posted Date: December 16th, 2020

DOl: https://doi.org/10.21203/rs.3.rs-124860/v1

License: (c) (i) This work is licensed under a Creative Commons Attribution 4.0 International License. Read Full License

Version of Record: A version of this preprint was published at Nature on June 22nd, 2022. See the published version at https://doi.org/10.1038/s41586-022-04884-x. 


\section{Abstract}

The regenerative potential of mammalian peripheral nervous system (PNS) neurons after injury is critically limited by their slow axonal regenerative rate ${ }^{1}$. Since a delayed target re-innervation leads to irreversible loss of function of target organs ${ }^{2}$, accelerated axonal regeneration is required to enhance functional outcomes following injury. Regenerative ability is influenced by both injury-dependent and injury-independent mechanisms ${ }^{3}$. Among the latter, environmental factors such as exercise and environmental enrichment have been shown to affect signalling pathways that promote axonal regeneration ${ }^{4}$. Several of these pathways, including modifications in gene transcription and protein synthesis, mitochondrial metabolism and release of neurotrophins, can be activated by intermittent fasting (IF $)^{5,6}$. IF has in turn been shown to increase synaptic plasticity ${ }^{7,8}$ and neurogenesis ${ }^{9}$, partially sharing molecular mechanisms with axonal regeneration. However, whether IF influences the axonal regenerative ability remains to be investigated. Here we show that IF promotes axonal regeneration after sciatic nerve crush in the mouse via an unexpected mechanism that relies upon the gram + gut microbiome and an increase of the gut bacteria-derived metabolite indole-3-propionic acid (IPA) in the serum. IPA production by Clostridium sporogenes is required for efficient axonal regeneration, and delivery of IPA after sciatic injury significantly enhances axonal regeneration, accelerating recovery of sensory function. Mechanistically, RNA sequencing analysis from sciatic dorsal root ganglia suggested a role for neutrophil chemotaxis in the IPA-dependent regenerative phenotype that was confirmed by the inhibition of neutrophil chemotaxis. Our results demonstrate for the first time the ability of a microbiome derived metabolite, such as IPA, in facilitating regeneration and functional recovery of sensory axons via an immune-mediated mechanism. Since IPA is a naturally occurring metabolite with a very favourable toxicity profile, it represents a realistic translational possibility for human axonal injuries.

\section{Main}

Injuries to the peripheral nervous system (PNS) have a high prevalence with millions of affected individuals worldwide and are typically followed by long-lasting neurological disability without effective treatments beyond surgical reconstruction, which is only effective in a small percentage of cases ${ }^{10-12}$. These injuries frequently result in partial or total loss of sensory, motor and autonomic function due to inefficient and slow axonal regeneration ${ }^{13,14}$. The study of cellular and molecular responses to injury ${ }^{15,16}$, of neurodevelopmental pathways ${ }^{17,18}$, of regenerative organisms ${ }^{18,19}$, as well as molecular screening approaches ${ }^{20}$ have contributed to the identification of a number of mechanisms that influence regenerative ability. However, our knowledge of the cellular and molecular mechanisms underpinning axonal regeneration and functional recovery remains incomplete, undermining the development of effective treatments for clinical injuries.

Recently, accumulating evidence implicates healthy lifestyle choices such as exercise and environmental enrichment in priming and enhancing the regenerative potential of sensory neurons ${ }^{4}$. Similarly, dietary regimens including intermittent fasting (IF) have been shown to promote metabolic and signalling 
pathways with the potential to favour wound repair and recovery in several disease states, including following nervous system injury ${ }^{21-24}$.

Therefore, we investigated whether IF would prime sensory neurons for enhanced axonal regeneration and recovery in a model of sciatic nerve injury and whether it would enable the identification of specific molecular mechanisms with translational potential for nerve repair.

\section{Intermittent fasting promotes axonal regeneration following sciatic nerve crush via the gram + gut microbiome}

Axonal regeneration was initially assessed 24 hours after a sciatic nerve crush (SNC) following 10 or 30 days of IF versus an ad libitum (AL) diet. Both 10 and 30 days IF regimens significantly enhanced regeneration past the crush site to a similar extent (Extended data Fig. 1a-c), therefore all subsequent experiments were performed following 10 days of IF. Importantly, IF also promoted axonal regeneration when the recovery time was extended to 72 hours after SNC (Fig. 1a-C). In confirmation of these findings, IF was followed by an increase in neurite outgrowth in cultured dorsal root ganglia (DRG) neurons (Extended data Fig. 1d-f). Importantly, IF did not alter critical modifiers of axonal regeneration such as Schwann cell and macrophage recruitment ${ }^{25,26}$ to the nerve crush site (Extended data Fig. 2a-d) or the neurotrophic factors BDNF, NGF, NT3 and NT4/5 in DRG ${ }^{27-31} 72$ h post-SNC (Extended data Fig. 2e), suggesting that Schwann cells, macrophages and neurotrophic factors likely do not play a central role in the IF-dependent regenerative phenotype.

Since IF modifies cell metabolism ${ }^{32,33}$ it was hypothesised that changes in metabolism may underpin the IF-dependent regenerative ability. To this end, semi-targeted gas chromatography coupled to mass spectrometry (GC-MS) of extracted serum following IF vs AL was performed, enabling identification of 79 metabolites, 14 of which were differentially enriched upon IF (Fig. 1d-f, Extended data Fig. 3). These metabolites could be clustered into 2 groups: microbiome derived and host metabolites (Fig. 1e). Interestingly, the 4 most enriched metabolites upon IF were the microbiome derived 3-indolelactic acid 2, 2,3-butanediol 2, xylose 2 and indole-propionic acid (IPA) (Fig. 1f). To assess whether the IF-dependent microbiome promotes sciatic nerve regeneration, microbiota collected from IF fed animals were transplanted via faecal transplantation (FT) into AL mice: indeed, FT from IF-mice led to a significant increase in regenerating fibres past the crush site in AL mice after SNC (Extended data Fig. 4). Since indole metabolites are primarily synthesized from tryptophan by the gram-positive bacteria Bifidobacterium (indole-3-lactic acid) ${ }^{34}$, Lactobacillus and Clostridium sporogenes (indole-propionic acid $)^{35}$, the requirement of gram + bacteria for IF-dependent axonal regeneration was investigated. Vancomycin treatment, which depletes gram + bacteria, abolished IF-dependent axonal regeneration (Fig. 2a-c), indicating that gram + bacteria are required for IF-dependent regeneration.

To identify metabolites underpinning the microbiome-dependent regenerative phenotype, serum samples were profiled by GC-MS following IF vs AL with and without vancomycin (Fig. 2d-k, Extended data Fig. 5). Remarkably, IPA was the only metabolite significantly affected by vancomycin treatment with the most 
significant interaction p-value, suggesting that its amount critically depended on IF and the presence of the gram + gut microbiome (Fig. 2e, Table 1). Indeed, following IF, serum IPA was found among the highest increased metabolites, which was completely depleted by vancomycin (Fig. 2f), as confirmed by targeted Liquid Chromatography-tandem mass spectrometry (LC-MS/MS) (Fig. 2g, h). As expected, vancomycin significantly reduced bacterial diversity as shown by $16 \mathrm{~S}$ rDNA sequencing (Extended data Fig. 6a, b, Supp File 1 and 2) and decreased the number of Bacteroidetes and Firmicutes, which were increased after IF (Extended data Fig. 6c). Importantly, the vast majority of Firmicutes belongs to the order of Clostridiales (Extended data Fig. 6d), and the top 5 most increased bacteria following IF are also Clostridiales (Extended data. Figure 6e), confirming Vancomycin treatment impacts IF- dependent pathways.

Together, these data suggest that gram + bacteria producing IPA are responsible for IF dependent axonal regeneration.

\section{IPA promotes axonal regeneration after sciatic nerve crush via neutrophil chemotaxis and IFNY}

Next, it was investigated whether IPA synthesis by the gut microbiome is required for axonal regeneration after SNC. Following vancomycin-mediated depletion of gram + bacteria in mice, the gut was recolonised with either a mutant Clostridium sporogenes fldC (C.s. fldC) bacterial strain that cannot produce IPA ${ }^{36}$ or with a C.s. WT strain and axonal regeneration was measured 72 hours after a SNC (Fig. 3a-d). Depletion of IPA from the serum of mice recolonised with the C.s. fldC (Fig. 3b) resulted in significantly reduced axonal regeneration compared with the C.s. WT (Fig. 3c, d). Conversely, IPA gavage significantly and robustly increased axonal regeneration of the sciatic nerve (Fig. 3e-h).

Together these studies demonstrate that bacterial production of IPA in the gut critically affects axonal regeneration. Since changes in IPA production in the gut are reflected by variations in the serum, IPA was administered by intraperitoneal (i.p.) injection and axonal regeneration was assessed 72 hours after SNC. Remarkably, systemic IPA delivery mirrored the increase in axonal regeneration elicited by IPA gavage (Extended data Fig. 7a-c), indicating that serum IPA might affect DRG neuron growth either directly or indirectly via neuronal intrinsic or extrinsic mechanisms respectively.

When IPA was administered directly into the media of cultured DRG neurons no changes in neurite outgrowth were observed (Extended data Fig. 7d-f), suggesting that IPA affects growth via an extrinsic regenerative mechanism to the DRG neurons.

To investigate IPA-dependent molecular signatures potentially affecting the regenerative ability of DRG neurons, RNA sequencing was performed from DRG preceding (naïve) or 72 hours after SNC following IPA or PBS oral gavage. IPA treatment affected the DRG gene expression programme as shown by differential gene expression analysis (Extended data Fig. 8a-c, Supp File 3 and 4), increased the expression of the gene encoding for the IPA nuclear receptor Pregnane X Receptor (Nr1i2), supporting activation of IPA signalling, and it affected mainly genes related to immune-regulatory GO categories, with the most significant enrichment for "neutrophil chemotaxis" at 72 hours following SNC (Fig. 4a, b, 
Supp File 4 and 5). Notably, the neutrophil ligand Cd177 and endothelial neutrophil chemoattractant chemokine (C-X-C motif) ligand 1 (Cxcl1) were selectively upregulated by IPA after SNC (Extended data Fig. 8c), suggesting that IPA might promote neutrophil chemotaxis towards the DRG via a CXCR2 mediated mechanism.

In support of this model, we found that IPA treatment increased the number of neutrophils within the DRG tissue, but not at the nerve crush site (Extended data Fig. 9a-h). The number of other immune cells including CD4 and CD8 T-cells, macrophages, NK and B-cells, did not differ between IPA and vehicle (Extended data Fig. 10). DRG immunolabelling showed that the majority of neutrophils were positive for CXCR2 (Extended data Fig. 9i, j), a marker of neutrophil activation and chemotaxis, suggesting that they might be activated and attracted by a CXCR2 mediated mechanism. To test this hypothesis, neutrophil chemotaxis was inhibited at the time of IPA treatment by using an anti-CXCR2 neutralising monoclonal antibody that significantly impaired IPA-dependent regeneration after SNC (Fig. 4c-g, Extended data Fig. 11j-k). Accordingly, neutrophil depletion with anti-Ly6G monoclonal antibody significantly decreased axonal regeneration mediated by IPA compared with IgG control antibody (Extended Data Fig. 11a-i). Together these data indicate that IPA-dependent neutrophil chemotaxis is required for axonal regeneration after SNC.

RNA sequencing also identified an IPA-dependent increase in IFNy signalling in the DRG, indicating a potential signalling mechanism downstream of neutrophil recruitment (Extended data Fig. 12a). Indeed, monoclonal antibody mediated neutralisation of IFNy significantly reduced IPA-dependent sciatic nerve regeneration (Extended data Fig. 12b-c). In line with these data, IFNY treatment in cultured DRG neurons and in vivo IFNy delivery followed by ex vivo analysis of DRG neuronal outgrowth in culture revealed a significant increase in regenerative growth (Extended data Fig. 12d-g).

\section{IPA delivery after sciatic nerve crush injury improves sensory neurological recovery and epidermal innervation}

Next, whether IPA administration at a clinically suitable time 24 hours after injury was able to accelerate the rate of sensory recovery following sciatic nerve injury was investigated. Both recovery of physiological sensory function by assessing responses to thermal stimulation and the presence of mechanical allodynia, which can be an undesired consequence of maladaptive epidermal re-innervation, were evaluated (Fig. 4h-k). IPA treated mice showed a faster speed of recovery of thermal nociception as shown by the Hargreaves test, displaying a reduced withdrawal response time compared to PBS treated mice between day 7 and day 19 (Fig. 4j), however, no changes in mechanical allodynia, measured with Von Frey filaments, between IPA- and PBS treated groups were observed (Fig. 4k). These data show that IPA leads to an accelerated recovery in thermal nociception while it does not induce mechanical allodynia following sciatic nerve injury. Importantly, anti-PGP9.5 immunostaining of the hind paw interdigital skin at 16 days post-SNC revealed a significantly increased epidermal innervation following IPA vs PBS, providing an anatomical substrate for functional recovery (Fig. 4I-n). Together, these experiments indicate 
that IPA induces sensory recovery including by improving epidermal innervation without causing neuropathic pain in a sciatic crush model of peripheral nerve injury.

\section{Conclusions}

The present study identifies a novel dietary-dependent regenerative mechanism that relies on the gut gram + Clostridiales bacterial production of the metabolite IPA. IPA accelerates axonal regeneration, epidermal innervation and sensory neurological recovery after SNC via the recruitment of neutrophils to the DRG. The gut microbial metabolite IPA, a product of bacterial metabolism of tryptophan in the lower gut, and its xenobiotic nuclear receptor PXR have been implicated in a variety of immune responses in several tissues and cell types ${ }^{37-41}$, including in the gut microenvironment, where an immune suppressive and anti-inflammatory function for IPA and PXR signalling have been described ${ }^{37-39}$. Conversely, PXR activation in vascular endothelial cells has been shown to initiate an innate inflammatory response ${ }^{41}$, which results in neutrophil recruitment ${ }^{42}$, supporting our model that suggests an IPA-dependent increase of trans-endothelial neutrophil chemotaxis.

While neutrophils are required for axonal regeneration in the $\mathrm{PNS}^{43}$ and $\mathrm{CNS}^{44-46}$ via changes in their recruitment at the lesion site, our study supports a regenerative role for neutrophils by communicating regenerative signals such as IFNY at the level of the DRG where the neuronal cell bodies reside.

IFNy-dependent axonal regeneration is in line with increased inflammatory signalling by neutrophils, however, a role for additional cytokines supporting IPA-dependent axonal regeneration cannot be ruled out.

Since the gut microbiome undergoes profound changes in human subjects upon nervous system injury ${ }^{47}$, identifying gut microbiome derived regenerative small molecules for oral or intravenous administration, could provide promising new therapeutic possibilities to heal nerve injury, promote axonal regeneration and enhance neurological recovery.

\section{Methods}

\section{Animal Husbandry}

All animal experiments were carried out according to UK Home Office regulations in line with the Animals (Scientific Procedures) Act of 1986 under personal and project licenses registered with the UK Home Office. Mice were maintained in-house under standard housing conditions (12-h light/dark cycles), $24 \mathrm{hr}$ access to water and standard chow diet. All mice were of a C57BL6 background (male, 20-30 g, 6-8 weeks of age). Experimenters were blinded to experimental groups during scoring and quantifications. Sample sizes were chosen in accordance with similar previously published experiments.

\section{Intermittent Fasting}


Body weight-matched 6-8 week old male C57BL/6 mice were randomly assigned to intermittent fasting (IF), or ad libitum (control) treatment groups. The IF group did not have access to food (fasting) during the first $24 \mathrm{~h}$ and then every second day after that (e.g. fasting during 0-24 h, 48-72 h, 96-120 h) with ad libitum access to food on the alternating days (24-48 h, 72-96 h, 120-144 h). Pre-weighed food was provided in the food hopper of their home cage at 9:30 am (unless a fasting day for the IF groups), and leftover food was weighed $24 \mathrm{~h}$ later.

\section{Sciatic nerve crush (SNC) surgery}

Mice were anesthetized with isoflurane ( $5 \%$ induction, $2 \%$ maintenance), shaved on the hind limbs and lower back, sterilised with iodine, and an ophthalmic solution was applied to the eyes to prevent drying. An incision was made on the skin and the biceps femoris and the gluteus superficialis were opened by blunt dissection and the sciatic nerve was exposed using a surgical hook. The sciatic nerve crush was performed orthogonally for 20 seconds ( 45 seconds for reinnervation experiment) using a $5 \mathrm{~mm}$ surgery forceps (91150-20 Inox-Electronic). The crush was performed at approximately $20 \mathrm{~mm}$ distally from the sciatic DRG.

\section{Faecal transplantation}

Fresh faeces were collected every day from the cage of 5 healthy C57BL/ 6 mice, approximately $200 \mathrm{mg} /$ mouse. A fresh cage was used each day.. $1 \mathrm{~g}$ of faeces was then homogenized in $10 \mathrm{ml}$ of PBS for 30 seconds at $3000 \mathrm{rpm}$ at $4^{\circ} \mathrm{C}$. Thereafter, the supernatant was transferred to a new tube and centrifuged for $5 \mathrm{~min}$ at $12000 \mathrm{rpm}$ at $4^{\circ} \mathrm{C}$. The pellet was resuspended in $2.5 \mathrm{ml}$ PBS and $500 \mu \mathrm{l}$ gavaged to each mouse.

\section{Antibiotic administration}

Vancomycin (Sigma, V2002) was administered through the drinking water (50mg/kg/day; $214.27 \mathrm{mg} / \mathrm{l})$, which was replenished every second day.

\section{Indole-3-propionate treatment}

Indole-3-propionic acid (Sigma, 57400) was diluted at $0.5 \mathrm{mg} / 200 \mu \mathrm{l}$ in sterile PBS. Mice were treated with $20 \mathrm{mg} / \mathrm{kg} /$ day via gavage or IP injection.

\section{Clostridium sporogenes recolonization}

WT and $f l d C$ (mutant for (R)-phenyllactyl-CoA dehydratase beta subunit of the phenyllactate dehydratase complex [fldC]) Clostridium sporogenes were cultured overnight in trypticase yeast extract medium anaerobically. Bacterial cultures were mixed with glycerol to reach the final concentrations of: WT $6.01 \mathrm{E}+06 \mathrm{CFU} / \mathrm{ml}$ and C.s. fldC mutant $5.46 \mathrm{E}+06 \mathrm{CFU} / \mathrm{ml}$ in hermetically sealed glass vials. Mice were pre-treated with vancomycin in drinking water for 3 days. C.s. WT or C.s. were transplanted to the cecum via oral gavage for 10 consecutive days at $1 \mathrm{E}+06 \mathrm{CFU} /$ day. 


\section{Monoclonal Antibody injection}

Preceding and following nerve injury, monoclonal antibodies; mouse aLy6G (Bioxcell, Clone 1A8); mouse alFNy (Bioxcell, clone XMG1.1); rat IgG2a isotype control (Bioxcell, 2A3); rat algG1 (Bioxcell, clone HRPN); were administered at $200 \mu \mathrm{g} /$ day for 10 consecutive days via i.p. injection. Monoclonal antibodies antiCXCR2 (R\&D Systems, clone \# 242216) and anti-IgG2A (R\&D Systems, clone \# 54447) were administered for 3 consecutive days post nerve injury at $20 \mu \mathrm{g} /$ day via i.p. injection.

\section{Serum and cecum preparation}

Blood was extracted via heart punctuation and collected into a covered test tube. The blood was allowed to clot for a minimum of $30 \mathrm{~min}$ at room temperature and centrifuged at $2000 \mathrm{~g}$ for $10 \mathrm{~min}$ at $4{ }^{\circ} \mathrm{C}$. The supernatant was then collected into a fresh tube and stored at $-20^{\circ} \mathrm{C}$ until use.

Cecum content was removed from the cecum using sterile forceps into a tube and snap-frozen in liquid nitrogen immediately.

\section{Gas chromatography - Mass Spectrometry (GC-MS) untargeted metabolomics}

Serum samples $(100 \mu \mathrm{l})$ were prepared as follows: I) samples were spiked with $10 \mu \mathrm{L}$ internal standard solution (myristic acid-d27, 750mg/ml), II) $850 \mu \mathrm{L}$ of ice cold methanol were added, followed by centrifugation for $20 \mathrm{~min}\left(4^{\circ} \mathrm{C}, 16000 \mathrm{~g}\right)$, III) $750 \mu \mathrm{L}$ of supernatants were transferred to silanized dark $2 \mathrm{~mL}$ autosampler vials and were evaporated to dryness in a rotational vacuum concentrator $\left(45^{\circ} \mathrm{C}, 20\right.$ mbar, $2 \mathrm{~h}$ ), IV) $50 \mu \mathrm{L}$ of methoxyamine solution ( $2 \%$ in pyridine) were added and the samples were incubated overnight at room temperature and finally, V) $100 \mu \mathrm{L}$ of N-methyl-trimethylsilyltrifluoroacetamide (MSTFA) $+1 \%$ trimethylchlorosilane (TMCS) solution were added, the samples were incubated at $60^{\circ} \mathrm{C}$ for $1 \mathrm{~h}$ and were transferred to dark autosampler vials with $250 \mu \mathrm{L}$ silanized inserts. The samples were analysed in an Agilent 7890B-5977B Inert Plus GC-MS system. $2 \mu \mathrm{L}$ of each sample were injected in a split inlet (1:10 ratio). The chromatographic column was an Agilent ZORBAX DB5- MS (30 m X $250 \mu \mathrm{m} \times 0.25 \mu \mathrm{m}+10 \mathrm{~m}$ Duraguard). The temperature gradient was $37.5 \mathrm{~min}$ long and the mass analyser was operated in full scan mode between $50-600 \mathrm{~m} / \mathrm{z}$. The detailed instrumental conditions are described elsewhere (Agilent G1676AA Fiehn GC/MS Metabolomics RTL Library, User Guide, Agilent Technologies, https://www.agilent.com/cs/library/usermanuals/Public/G167690001_Fiehn.pdf). Quality Control (QC) samples were created by pooling equal amounts of every serum sample of the study and were analysed interspaced in the analytical run. Study samples were randomized before sample preparation. Peak deconvolution, alignment and annotation and were performed with the use of the Fiehn library via the software packages AMDIS (NIST), Mass Profiler Pro and Unknowns (Agilent technologies) in the pooled QC samples. Peak picking was performed with the GAVIN package (A software complement to AMDIS for processing GC-MS metabolomics data. doi: 10.1016/j.ab.2011.04.009) ${ }^{48}$. Non-reproducible (CV>30\% in QC samples) and contaminated (blank > 20\% of the mean QC levels) metabolic features were removed from the dataset. 
This data set was then used to build orthogonal partial least-squares-discriminant analysis (OPLS-DA) (IF vs $A L$ ) or partial least-squares-discriminant analysis (PLS-DA) (IF, IF-V, AL, AL-V), focusing on the differences among the experimental groups. The OPLS algorithm derives from the partial least-squares (PLS) regression method ${ }^{49}$. The method explains the maximum separation between class samples $Y$ ( $n$ dummy variables for $\mathrm{n}$ classes) by using the GC-MS data X. Here the ropls R package (http://bioconductor.org/packages/release/bioc/html/ropls.html) was used, which implements the PCA, PLS-DA and OPLS-DA approaches ${ }^{50}$. It includes $\mathrm{R}^{2}$ and $\mathrm{Q}^{2}$ goodness-of-fit and goodness-of-prediction statistics, permutation tests, as well as scores, loadings, predictions, diagnostics, outliers graphics ${ }^{50}$. R2X describes the percentage of predictive and orthogonal variation in $X$ that is explained by the full model. R2Y describes the total sum of variation in $Y$ explained by the model. Q2 describes the predictive performance of the model calculated by full cross validation.

\section{Liquid chromatography-tandem mass spectrometry (LC-MS/MS)}

\section{Materials}

Indole-3-propionic acid (Aldrich $1 \mathrm{~g}$ ) as standard and indole-3-propionic-2,2-d 2 acid (0.05 g, C.D.N. Isotopes) as internal standard (IS) were used. Acetonitrile, methanol and formic acid were of ULC-MS grade was supplied from Bio-Lab. Water with resistivity 18.2 M $\Omega$ was obtained using Direct 3-Q UV system (Millipore). Standard curve was built using concentration range of 3-indole propionic acid 0.01-12 $\mu \mathrm{g} / \mathrm{mL}$, with final concentration of IS $100 \mathrm{ng} / \mathrm{mL}$.

\section{Extract preparation}

Plasma $(30 \mu \mathrm{L})$ and IS (10uL, $1 \mathrm{ug} / \mathrm{mL})$ was incubated $10 \mathrm{~min}$, then $500 \mu \mathrm{L}$ of methanol was added. The mixture was shaken at $10^{\circ} \mathrm{C}$ for $30 \mathrm{~min}$ (ThermoMixer C, Eppendorf), and centrifuged at $21,000 \mathrm{~g}$ for 10 min. Collected supernatant was evaporated in speedvac and then in lyophilizer. Before LC-MC analysis, the obtained residue was re-suspended in $100 \mu \mathrm{L}$ of $20 \%$-aq methanol, centrifuged twice at $21,000 \mathrm{~g}$ for 5 min to remove insoluble material. The soluble part was placed to insert of LC-MS vial. Some samples were diluted $1 / 20$ with $20 \%$-aq methanol.

\section{$\underline{\text { LC-MS analysis }}$}

The LC-MS/MS instrument consisted of an Acquity I-class UPLC system (Waters) and Xevo TQ-S triple quadrupole mass spectrometer (Waters) equipped with an electrospray ion source and operated in positive ion mode. MassLynx and TargetLynx software (version 4.1, Waters) were applied for the acquisition and analysis of data. Chromatographic separation was done on a $100 \mathrm{~mm} \times 2.1 \mathrm{~mm}$ internal diameter, 1.7- $\mu \mathrm{m}$ UPLC BEH C18 column equipped with $50 \mathrm{~mm} \times 2.1 \mathrm{~mm}$ internal diameter, 1.7- $\mu \mathrm{m}$ UPLC BEH C18 pre-column (both Waters Acquity) with mobile phases $\mathrm{A}(0.01 \%$ formic acid) and $\mathrm{B}(0.01 \%$ formic acid in acetonitrile) at a flow rate of $0.6 \mathrm{ml} \mathrm{min}^{-1}$ and column temperature $60^{\circ} \mathrm{C}$. A gradient was used as follows: $0-5$ min a linear increase from 20 to $25 \% \mathrm{~B}$, then increase to $95 \%$ B during 0.1 min, 5.1-7 min held at $95 \%$ B, then back to $20 \%$ B in 0.1 min, and equilibration at $20 \%$ B for 1.9 min, 
providing total run time of $9 \mathrm{~min}$. Samples kept at $8{ }^{\circ} \mathrm{C}$ were automatically injected in a volume of $1 \mu \mathrm{l}$. MS parameters: capillary voltage - $1 \mathrm{kV}$, cone $0 \mathrm{~V}$, Source temperature $-150^{\circ} \mathrm{C}$, Desolvation temperature $550^{\circ} \mathrm{C}$, Desolvation gas - $110 \mathrm{~L} / \mathrm{Hr}$, Cone gas - $200 \mathrm{~L} / \mathrm{Hr}$, Nebulizer - 7.0 Bar, Collision gas $0.10 \mathrm{~mL} / \mathrm{min}$. MRM transitions (collision energy, eV): 190>55.0 (17), 190>130.1 (12), 190>172.1 (9) for 3-indole propionic acid, and 192.1>56.0 (12), 192.1>130.1 (14), 190>174.1 (14) for IS.

\section{S rDNA amplicon sequencing}

Cecum content was collected from mice and immediately frozen in liquid nitrogen. Samples were stored at $-80^{\circ} \mathrm{C}$. Faecal DNA was extracted using Fast DNA spin kit (MP Biomedicals). Libraries were prepared using the 16S Metagenomic Sequencing Library Preparation protocol for the Illumina MiSeq System. In brief, PCR primers (16S Amplicon PCR Forward Primer $=5^{\prime}$

TCGTCGGCAGCGTCAGATGTGTATAAGAGACAGCCTACGGGNGGCWGCAG; 16S Amplicon PCR Reverse Primer $=5{ }^{\prime}$ GTCTCGTGGGCTCGGAGATGTGTATAAGAGACAGGACTACHVGGGTATCTAATCC) directed at the V3/V4 region of bacterial rRNA genes were used to generate libraries and libraries were validated by the Agilent 2100 Bioanalyzer. Sequencing was performed with 250 bp paired-end reads on Illumina Miseq2500 (Imperial BRC Genomics Facility).

\section{$16 \mathrm{~S}$ rRNA amplicon sequencing data analysis}

Only single-end R1 sequences were analysed. Data processing was carried out in R statistical environment version 3.5.2 according to the DADA2 pipeline ${ }^{51}$ as follows; primers were trimmed from demultiplexed sequences, amplicon sequence variants (ASVs) were generated using DADA2 package version 1.10.1, and taxonomic assignment was performed using the SILVA rRNA database version 132 [https://www.arb-silva.de/]. ASVs with no phylum assigned were excluded from analyses to yield a final number of $n=906$ annotated ASVs. Alpha and beta diversity analyses were carried out using phyloseq package version $1.26 .1^{52}$. Alpha diversity (Shannon Index) was calculated using raw counts ${ }^{53}$. Groups were compared using Mann Whitney U statistical tests. For beta diversity analyses, data was normalised by log-transformation with a pseudo count of +1 . Differential abundance of groups were compared in the DESeq2 package version 1.22.2 ${ }^{54}$ using the Wald test with Benjamini and Hochberg adjustments for multiple testing ( $p$ value set as 0.05 ). Alpha diversity is a measure of diversity or "microbial variation" within a (single) sample while beta diversity is a measure of diversity across (several) samples.

\section{RNA sequencing}

DRG were collected from animals that had undergone 10 days of IPA or PBS treatment and/or followed by sciatic nerve crush. Sciatic DRG were extracted $72 \mathrm{~h}$ after sciatic nerve crush or from naïve animals (surgeries were performed as described above) and collected into RNAlater. DRG were crushed with RNase free micro pestle and RNA was then immediately extracted using RNAeasy kit (Qiagen), according to manufacturer's guidelines. Residual DNA contamination was removed by treating the spin column with 40 units of RNase-free DNase I (Qiagen) for $15 \mathrm{~min}$ at $23^{\circ} \mathrm{C}$ prior to RNA elution. RNA concentrations and purity were verified for each sample following elution with the Agilent 2100 Bioanalyzer (Agilent). RNA 
with RIN factors above 8.5 were used for library preparation. cDNA libraries for each sample were generated by the Imperial BRC Genomics Facility using the TruSeq Sample Preparation Kit A (Illumina, San Diego CA) and sequenced using Illumina HiSeq 4000 (PE 2x75 bp) sequencing. Gene ontology (G0) was performed on differentially expressed genes with DAVID 6.8 (Database for Annotation, Visualization, and Integrated Discovery (http://david.abcc.ncifcrf.gov/)). Differentially expressed genes were selected using a threshold of $\mathrm{P}<0.05$ and $|1.5|<F C$ (fold change) or no FC cut-off. To identify IPA-dependent specific differentially expressed genes following SNC, we compared IPA-SNCvsPBS and PBS-SNCvsPBS groups and selected the uniquely up- or downregulated genes in the IPA-SNCvsPBS group.

\section{Sciatic nerve regeneration}

24 or 72 hours following the surgery, sciatic nerves were dissected and post-fixed in 4\% PFA, incubated at $4^{\circ} \mathrm{C}$ for $1 \mathrm{~h}$ and transferred into $30 \%$ sucrose for at least 3 days. Subsequently, the tissue was embedded and frozen in Tissue-Tek OCT and maintained at $-80^{\circ} \mathrm{C}$ until cut into $11 \mu \mathrm{m}$ sagittal sections. Tissue sections were immunostained for SCG10 (1:1000, rabbit, Novus) a marker for regenerating axons. The crush site was identified by deformation of the nerve and disruption of axons coinciding with highest SCG-10 intensity. The SCG-10 intensity was measured in $500 \mu \mathrm{m}$ intervals along the length of the nerve distal to sciatic nerve crush site. The intensity was normalised to the SCG-10 intensity before the crush site and plotted as fold-change. 4-6 sections per animal were analysed and imaged with a HWF1 - Zeiss Axio Observer with a Hamamatsu Flash 4.0 fast camera using 10x magnification.

\section{Dorsal Root Ganglia (DRG) cell culture.}

Glass coverslips were coated with $0.1 \mathrm{mg} / \mathrm{ml} \mathrm{PDL}$, washed and coated with mouse Laminin $2 \mathrm{ug} / \mathrm{ml}$ (Millipore) for 1-2 hours each previous to the start of the experiment. Sciatic DRG from adult animals were dissected and collected in Hanks balanced salt solution (HBSS) on ice. The DRG were transferred into a digest solution ( $5 \mathrm{mg} / \mathrm{ml}$ Dispase II (Sigma), $2.5 \mathrm{mg} / \mathrm{ml}$ Collagenase Type II (Worthington) and incubated in a $37^{\circ} \mathrm{C}$ water bath for $45 \mathrm{~min}$, with occasional shaking for 30 seconds. Thereafter, the DRG were washed and manually dissociated with a $1 \mathrm{ml}$ pipette in media containing $10 \%$ heat inactivated FBS (Invitrogen) and 1x B27 (Invitrogen) in F12:DMEM (Invitrogen). Pipetting was continued until DRG were fully dissociated and no clumps could be observed. Next, the cell suspension was spun down at 1000 rpm for 4 min and resuspended in culture media containing 1x B27 and Penicillin/Streptomycin in F12 : DMEM. 3500 cells were plated on each coverslip (laminin and PDL coated) and maintained in a humidified culture chamber with $5 \% \mathrm{CO} 2$ at $37^{\circ} \mathrm{C}$, for 12 hours before fixed with $4 \%$ PFA and immunostained.

Following cell plating, DRG were treated with indole-3-propionic acid ( $1 \mathrm{mM}, 10 \mathrm{mM}$ or $100 \mathrm{mM}$, Sigma 57400 ) or IFNg (5 ng/ml, $100 \mathrm{ng} / \mathrm{ml}$, Peprotech 250530). For IFNy-dependent ex-vivo DRG outgrowth, animals were injected intraperitoneally with $10 \mu \mathrm{g}$ IFNy/mouse 48 hours before DRG cell culture.

\section{Immunohistochemistry}


Immunohistochemistry on tissue sections was performed according to standard procedures. Tissue sections were rehydrated with PBS and blocked and permeabilized for 1 hour with either $8 \%$ bovine serum albumin (Sigma Aldrich) or 10\% normal goat serum (Abcam) containing 0.3\% PBS-TritonX-100. Secondly, the sections were incubated with anti-SCG10 (1:1000, Novus), anit-SOX10 (1:1000, Abcam), anti-CD68 (1:1000, Abcam), anti-Ly6G (1:500, BioxCell, clone 1A8), anti-CD8 (1:100, ThermoFisher, 14-0081-82), antiTuj1 (1:500, Novus, NB100-1612) anti-CD68 (1:200, Abcam, ab125212), anti-B220 (1:100, Biolegend, 103228), anti-PGP9.5 (1:200, Proteintech, 14730-1-AP), CD4 (1:100, BD Pharmingen, Clone RM4-5), NK1.1 (1:2000, Bioxcell, BE0038), Ly6G-FITC (1:100, Biolegend), CXCR2 (1:50, R\&D Systems) at room temperature over-night. The sections were washed three times with PBS, followed by incubation with Alexa Fluor conjugated goat secondary antibodies for 1 hour. All tissue sections were counterstained with DAPI (Molecular Probes) and cover slipped with moviol.

\section{Immunocytochemistry (ICC)}

Plated cells were fixed by incubation with cold $4 \%$ PFA for $15 \mathrm{~min}$. Thereafter, they were blocked and permeabilized for 1 hour with $0.3 \%$ TX100 in PBS containing $2 \%$ BSA. The primary antibody staining was performed using anti- $\beta$ III Tubulin (1:1000, mouse, Promega) in $0.1 \%$ TX100 in PBS containing $2 \%$ BSA, which O/N incubation at RT. The goat secondary antibody (Alexa) was diluted in $0.1 \%$ TX100 in PBS containing $2 \%$ BSA and cells were incubated for 1 hour. All cells were counterstained with DAPI.

\section{FACS}

Cells were isolated from spleens or lymph nodes by mashing using a $70 \mu \mathrm{m}$ cell strainer and a pestle and transferred into Media (RPMI media 1640 [Thermo Fisher scientific] $+2 \%$ Foetal calf serum $+1 \mathrm{x}$ Penicillin/Streptomycin $+1 x$ HEPES [Thermo Fisher scientific]). Spleen cells were centrifuged at $1200 \mathrm{rpm}$ for $6 \mathrm{~min}$ and resuspended in $1 \mathrm{ml}$ of Red blood cell lysis buffer Hybrid Max (Sigma) for $5 \mathrm{~min}$. Next, $9 \mathrm{ml}$ of media was added and centrifuged at $1200 \mathrm{rpm}$ for $6 \mathrm{~min}$. Spleen cells or lymph node (LN) cells were resuspended in Media and plated at $1 \times 10^{6}-2 \times 10^{6}$ cells per well in a 96 -well plate. The plate was centrifuged at $2000 \mathrm{rpm}$ for $1 \mathrm{~min}$ and $50 \mu \mathrm{l}$ of blocking stain (1:50 dilution Rat Serum [Sigma], 1:50 dilution TruStain FCX ${ }^{\mathrm{TM}}$ [anti-mouse CD16/32] [Biolegend] in FACS buffer [PBS supplemented with with $5 \%$ FCS, 2mM EDTA and $0.09 \%$ NaAzide]) was added. Cells were blocked for 30 min at $4{ }^{\circ} \mathrm{C}$ and subsequently washed three times with $140 \mu$ l of FACS buffer. Antibodies were added and cells were incubated for 30 mins in the dark. Next, cells were washed another 3 times and analysed on a BD LSR Fortessa X-20 (BD Biosciences, 4 laser). 


\begin{tabular}{llll} 
Marker (clone) & Colour & $\underline{\text { Dilution }}$ & Company. \\
\hline CD45 (30-F11) & V450 & $1: 100$ & BD Biosciences \\
\hline CD11b (M 1/70) & BV421 & $1: 200$ & Biolegend \\
\hline CD11c (3.9) & PECy7 & $1: 200$ & Biolegend \\
\hline Ly6G (1A8) & BV711 & $1: 200$ & Biolegend \\
\hline Ly6C (HK1.4 & FITC & $1: 200$ & Biolegend \\
\hline LIVE/DEAD & Aqua $(405 \mathrm{~nm})$ & $1: 100$ & Thermo Fisher Scientific \\
\hline CD115 & PE & $1: 50$ & BD Biosciences
\end{tabular}

\section{Microscopy}

Photomicrographs were taken with a Nikon Eclipse TE2000 microscope with an optiMOS scMOS camera using 10x or 20x resolution Zeiss Axio Observer with a Hamamatsu Flash 4.0 fast camera using 10x or 20x magnification. Confocal images were taken with a Leica TCS SP8 II confocal microscope at 40X or $60 X$ magnification and processed with the LAS-AM Leica software (Leica).

\section{Image Analysis for IHC and ICC}

Image analysis was conducted using ImageJ (Fiji) software. All analysis was performed by the same experimenter who was blinded to the experimental groups. DRG images were taken using a Nikon Eclipse TE2000 microscope with an optiMOS scMOS camera at either 10x or 20x magnification. Images were analysed by counting the number of cells per area, measuring the intensity against the background or calculating percentage of cells with positive staining.

For neurite length analysis between 15 and 20 images were taken per coverslip and analysed using Neuron J plugin for Image $\mathrm{J}$ software (Image $\mathrm{J}$ ). All analyses were performed in blind. Approximately 4560 cells were analysed per animal and condition.

\section{Statistical analysis}

Results are graphed as mean \pm SEM. Statistical analysis was carried out using GraphPad Prism 7 . Normally distributed data was evaluated using a two-tailed unpaired Student's t-test or a one-way ANOVA when experiments contained more than two groups. Dunnett multiple comparisons test or multiple comparison testing corrected by FDR with Benjamini and Hochberg were applied when appropriate. The two-way ANOVA, Tukey's or Sidak's test, was applied when two independent variables on one dependent variable were assessed. $A$ threshold level of significance was set at $P<0.05$. Significance levels were 
defined as follows: * $P<0.05 ; * \star P<0.01 ; * \star * P<0.001 ; * \star \star * ~ p<0.0001$. All data analysis was performed blind to the experimental group.

\section{Declarations}

\section{Competing interests}

The authors declare no competing interests.

\section{Author contributions}

E.S. designed, performed experiments, data analysis and wrote the manuscript; J.S.C. performed experiments and data analysis; L.L.G. performed experiments and data analysis; G.K. performed experiments and data analysis; L. Z. performed experiments and data analysis; G.C. performed experiments and data analysis; A.M. performed experiments and data analysis; A.B. performed experiment; A.B. performed data analysis; F.D.V. performed experiment; M.E.D. performed data analysis and edited the manuscript; J.S. provided experimental advise and edited the manuscript; D.D. provided experimental advise and edited the manuscript; S.D.G. designed experiments, provided funding and wrote the manuscript.

\section{Acknowledgments}

This work was supported by start-up funds from the Department of Brain Sciences, Imperial College London (SDG); Wings for Life (SDG); The Rosetrees Trust (SDG). The MED lab is funded by METACARDIS (HEALTH-F4-2012-305312) and the UK Medical Research Council (MRC grant MR/M501797/1). The research was supported by the National Institute for Health Research (NIHR) Imperial Biomedical Research Centre (MED, SDG). The views expressed are those of the author(s) and not necessarily those of the NHS, the NIHR or the Department of Health. We would like to thank Ilaria Palmisano for critically reading the manuscript and providing feedback.

\section{Data availability}

RNA sequencing

Accession GSE161342; https://www.ncbi.nlm.nih.gov/geo/query/acc.cgi?acc=GSE161342

Reviewer access token: ezclioqypfchtqr

$16 \mathrm{~S}$ rDNA amplicon sequencing 
Accession GSE162763; https://www.ncbi.nlm.nih.gov/geo/query/acc.cgi?acc=GSE162763

Reviewer access token: wbwrswekdxsprej

\section{References}

1. Scheib, J. \& Hoke, A. Advances in peripheral nerve regeneration. Nat Rev Neuro/9, 668-676, doi:10.1038/nrneurol.2013.227 (2013).

2. Lee, S. K. \& Wolfe, S. W. Peripheral Nerve Injury and Repair. JAAOS - Journal of the American Academy of Orthopaedic Surgeons 8 (2000).

3. Ferguson, T. A. \& Son, Y. J. Extrinsic and intrinsic determinants of nerve regeneration. J Tissue Eng 2, 2041731411418392, doi:10.1177/2041731411418392 (2011).

4. Hutson, T. H. et al. Cbp-dependent histone acetylation mediates axon regeneration induced by environmental enrichment in rodent spinal cord injury models. Science Translational Medicine 11, eaaw2064, doi:10.1126/scitransImed.aaw2064 (2019).

5. Mattson, M. P., Moehl, K., Ghena, N., Schmaedick, M. \& Cheng, A. Intermittent metabolic switching, neuroplasticity and brain health. Nat Rev Neurosci 19, 63-80, doi:10.1038/nrn.2017.156 (2018).

6. Longo, V. D. \& Mattson, M. P. Fasting: molecular mechanisms and clinical applications. Cell Metab 19, 181-192, doi:10.1016/j.cmet.2013.12.008 (2014).

7. Fontan-Lozano, A. et al. Caloric restriction increases learning consolidation and facilitates synaptic plasticity through mechanisms dependent on NR2B subunits of the NMDA receptor. J Neurosci 27, 10185-10195, doi:10.1523/JNEUROSCI.2757-07.2007 (2007).

8. Dasgupta, A., Kim, J., Manakkadan, A., Arumugam, T. V. \& Sajikumar, S. Intermittent fasting promotes prolonged associative interactions during synaptic tagging/capture by altering the metaplastic properties of the CA1 hippocampal neurons. Neurobiol Learn Mem, doi:10.1016/j.nlm.2017.12.004 (2017).

9. Lee, J., Seroogy, K. B. \& Mattson, M. P. Dietary restriction enhances neurotrophin expression and neurogenesis in the hippocampus of adult mice. J Neurochem 80, 539-547 (2002).

10. Asplund, M., Nilsson, M., Jacobsson, A. \& von Holst, H. Incidence of traumatic peripheral nerve injuries and amputations in Sweden between 1998 and 2006. Neuroepidemiology 32, 217-228, doi:10.1159/000197900 (2009).

11. Evans, G. R. Peripheral nerve injury: a review and approach to tissue engineered constructs. Anat Rec 263, 396-404, doi:10.1002/ar.1120 (2001).

12. Taylor, C. A., Braza, D., Rice, J. B. \& Dillingham, T. The Incidence of Peripheral Nerve Injury in Extremity Trauma. American Journal of Physical Medicine \& Rehabilitation 87, 381-385, doi:10.1097/PHM.0b013e31815e6370 (2008).

13. Afsoun Seddighi, A. N., Amir Saied Seddighi, Ali Reza Zali, Seyed Mahmood Tabatabaei, Ali Reza Sheykhi, Fatemeh Yourdkhani, Shoayb Naeimian. Peripheral Nerve Injury: A Review Article.. 
International Clinical Neuroscience Journal 3, 6 (2016).

14. Li, R. et al. Peripheral nerve injuries treatment: a systematic review. Cell Biochem Biophys 68, 449454, doi:10.1007/s12013-013-9742-1 (2014).

15. Mahar, M. \& Cavalli, V. Intrinsic mechanisms of neuronal axon regeneration. Nat Rev Neurosci 19, 323-337, doi:10.1038/s41583-018-0001-8 (2018).

16. Lindborg, J. A. et al. Molecular and cellular identification of the immune response in peripheral ganglia following nerve injury. J Neuroinflammation 15, 192, doi:10.1186/s12974-018-1222-5 (2018).

17. Strand, N. S. et al. Wnt/beta-catenin signaling promotes regeneration after adult zebrafish spinal cord injury. Biochem Biophys Res Commun 477, 952-956, doi:10.1016/j.bbrc.2016.07.006 (2016).

18. Ghosh, S. \& Hui, S. P. Axonal regeneration in zebrafish spinal cord. Regeneration (Oxf) 5, 43-60, doi:10.1002/reg2.99 (2018).

19. Shimizu, Y., Ueda, Y. \& Ohshima, T. Wnt signaling regulates proliferation and differentiation of radial glia in regenerative processes after stab injury in the optic tectum of adult zebrafish. Glia 66, 13821394, doi:10.1002/glia.23311 (2018).

20. Chandran, V. et al. A Systems-Level Analysis of the Peripheral Nerve Intrinsic Axonal Growth Program. Neuron 89, 956-970, doi:10.1016/j.neuron.2016.01.034 (2016).

21. Fann, D. Y. et al. Intermittent fasting attenuates inflammasome activity in ischemic stroke. Exp Neurol 257, 114-119, doi:10.1016/j.expneurol.2014.04.017 (2014).

22. Fann, D. Y., Ng, G. Y., Poh, L. \& Arumugam, T. V. Positive effects of intermittent fasting in ischemic stroke. Exp Geronto/ 89, 93-102, doi:10.1016/j.exger.2017.01.014 (2017).

23. Jeong, M. A. et al. Intermittent fasting improves functional recovery after rat thoracic contusion spinal cord injury. J Neurotrauma 28, 479-492, doi:10.1089/neu.2010.1609 (2011).

24. Plunet, W. T. et al. Dietary restriction started after spinal cord injury improves functional recovery. Exp Neurol 213, 28-35, doi:10.1016/j.expneurol.2008.04.011 (2008).

25. Hervera, A. et al. Reactive oxygen species regulate axonal regeneration through the release of exosomal NADPH oxidase 2 complexes into injured axons. Nat Cell Biol 20, 307-319, doi:10.1038/s41556-018-0039-x (2018).

26. Poplawski, G. et al. Schwann cells regulate sensory neuron gene expression before and after peripheral nerve injury. Glia, doi:10.1002/glia.23325 (2018).

27. Lindsay, R. M. Nerve growth factors (NGF, BDNF) enhance axonal regeneration but are not required for survival of adult sensory neurons. J Neurosci 8, 2394-2405 (1988).

28. Hollis, E. R., 2nd, Jamshidi, P., Löw, K., Blesch, A. \& Tuszynski, M. H. Induction of corticospinal regeneration by lentiviral trkB-induced Erk activation. P Natl Acad Sci USA 106, 7215-7220, doi:10.1073/pnas.0810624106 (2009).

29. Liu, Y. et al. NT-3 promotes proprioceptive axon regeneration when combined with activation of the mTor intrinsic growth pathway but not with reduction of myelin extrinsic inhibitors. Exp Neuro/ 283, 
73-84, doi:10.1016/j.expneurol.2016.05.021 (2016).

30. Boyd, J. G. \& Gordon, T. Neurotrophic Factors and Their Receptors in Axonal Regeneration and Functional Recovery After Peripheral Nerve Injury. Molecular neurobiology 27, 277-324, doi:10.1385/MN:27:3:277 (2003).

31. Hoke, A. et al. Schwann cells express motor and sensory phenotypes that regulate axon regeneration. J Neurosci 26, 9646-9655, doi:10.1523/JNEUROSCI.1620-06.2006 (2006).

32. Anson, R. M. et al. Intermittent fasting dissociates beneficial effects of dietary restriction on glucose metabolism and neuronal resistance to injury from calorie intake. P Natl Acad Sci USA 100, 62166220, doi:DOI 10.1073/pnas.1035720100 (2003).

33. Patterson, R. E. \& Sears, D. D. Metabolic Effects of Intermittent Fasting. Annu Rev Nutr, doi:10.1146/annurev-nutr-071816-064634 (2017).

34. Aragozzini, F., Ferrari, A., Pacini, N. \& Gualandris, R. Indole-3-lactic acid as a tryptophan metabolite produced by Bifidobacterium spp. Applied and Environmental Microbiology 38, 544-546 (1979).

35. Zhang, L. S. \& Davies, S. S. Microbial metabolism of dietary components to bioactive metabolites: opportunities for new therapeutic interventions. Genome Med 8, 46, doi:10.1186/s13073-016-0296-x (2016).

36. Dodd, D. et al. A gut bacterial pathway metabolizes aromatic amino acids into nine circulating metabolites. Nature 551, 648-652, doi:10.1038/nature24661 (2017).

37. Qiu, Z. et al. Pregnane X Receptor Regulates Pathogen-Induced Inflammation and Host Defense against an Intracellular Bacterial Infection through Toll-like Receptor 4. Sci Rep 6, 31936, doi:10.1038/srep31936 (2016).

38. Venkatesh, M. et al. Symbiotic bacterial metabolites regulate gastrointestinal barrier function via the xenobiotic sensor PXR and Toll-like receptor 4. Immunity 41, 296-310, doi:10.1016/j.immuni.2014.06.014 (2014).

39. Alexeev, E. E. et al. Microbiota-Derived Indole Metabolites Promote Human and Murine Intestinal Homeostasis through Regulation of Interleukin-10 Receptor. Am J Pathol 188, 1183-1194, doi:10.1016/j.ajpath.2018.01.011 (2018).

40. Hudson, G. et al. Pregnane X Receptor Activation Triggers Rapid ATP Release in Primed Macrophages That Mediates NLRP3 Inflammasome Activation. J Pharmacol Exp Ther 370, 44-53, doi:10.1124/jpet.118.255679 (2019).

41. Wang, S. et al. Xenobiotic pregnane $X$ receptor (PXR) regulates innate immunity via activation of NLRP3 inflammasome in vascular endothelial cells. J Biol Chem 289, 30075-30081, doi:10.1074/jbc.M114.578781 (2014).

42. Biondo, C. et al. The interleukin-1beta/CXCL1/2/neutrophil axis mediates host protection against group B streptococcal infection. Infect Immun 82, 4508-4517, doi:10.1128/IAI.02104-14 (2014).

43. Lindborg, J. A., Mack, M. \& Zigmond, R. E. Neutrophils Are Critical for Myelin Removal in a Peripheral Nerve Injury Model of Wallerian Degeneration. J Neurosci 37, 10258-10277, doi:10.1523/JNEUROSCI.2085-17.2017 (2017). 
44. Stirling, D. P., Liu, S., Kubes, P. \& Yong, V. W. Depletion of Ly6G/Gr-1 leukocytes after spinal cord injury in mice alters wound healing and worsens neurological outcome. J Neurosci 29, 753-764, doi:10.1523/JNEUROSCI.4918-08.2009 (2009).

45. Kurimoto, T. et al. Neutrophils express oncomodulin and promote optic nerve regeneration. $J$ Neurosci 33, 14816-14824, doi:10.1523/JNEUROSCI.5511-12.2013 (2013).

46. Sas, A. R. et al. A new neutrophil subset promotes CNS neuron survival and axon regeneration. Nat Immuno/ 21, 1496-1505, doi:10.1038/s41590-020-00813-0 (2020).

47. Kigerl, K. A. et al. Gut dysbiosis impairs recovery after spinal cord injury. J Exp Med 213, 2603-2620, doi:10.1084/jem.20151345 (2016).

48. Behrends, V., Tredwell, G. D. \& Bundy, J. G. A software complement to AMDIS for processing GC-MS metabolomic data. Anal Biochem 415, 206-208, doi:10.1016/j.ab.2011.04.009 (2011).

49. Trygg, J. \& Wold, S. Orthogonal projections to latent structures (O-PLS). Journal of Chemometrics 16, 119-128, doi:10.1002/cem.695 (2002).

50. Thévenot, E. A., Roux, A., Xu, Y., Ezan, E. \& Junot, C. Analysis of the Human Adult Urinary Metabolome Variations with Age, Body Mass Index, and Gender by Implementing a Comprehensive Workflow for Univariate and OPLS Statistical Analyses. Journal of Proteome Research 14, 3322-3335, doi:10.1021/acs.jproteome.5b00354 (2015).

51. Callahan, B. J. et al. DADA2: High-resolution sample inference from Illumina amplicon data. Nat Methods 13, 581-583, doi:10.1038/nmeth.3869 (2016).

52. McMurdie, P. J. \& Holmes, S. phyloseq: an R package for reproducible interactive analysis and graphics of microbiome census data. PLoS One 8, e61217, doi:10.1371/journal.pone.0061217 (2013).

53. McMurdie, P. J. \& Holmes, S. Waste not, want not: why rarefying microbiome data is inadmissible. PLoS Comput Biol 10, e1003531, doi:10.1371/journal.pcbi.1003531 (2014).

54. Love, M. I., Huber, W. \& Anders, S. Moderated estimation of fold change and dispersion for RNA-seq data with DESeq2. Genome Bio/ 15, 550, doi:10.1186/s13059-014-0550-8 (2014).

\section{Tables}




\begin{tabular}{|l|c|c|c|c|c|}
\hline Metabolite & log FC & \multicolumn{3}{|c|}{ p-value } & Rsq \\
\hline & IF vs AL & IF vs AL & Ab vs non-Ab & interaction & \\
\hline indole-3-propionic acid & 0.83 & $1.11 \mathrm{E}-05$ & $4.26 \mathrm{E}-06$ & $9.07 \mathrm{E}-04$ & $7.94 \mathrm{E}-01$ \\
\hline 3-indolelactic acid & 1.47 & $2.73 \mathrm{E}-05$ & $7.67 \mathrm{E}-02$ & $2.50 \mathrm{E}-01$ & $4.50 \mathrm{E}-01$ \\
\hline xylose & 1.11 & $3.47 \mathrm{E}-04$ & $1.72 \mathrm{E}-01$ & $8.79 \mathrm{E}-03$ & $4.74 \mathrm{E}-01$ \\
\hline 2,3-butanediol & 1.37 & $3.03 \mathrm{E}-04$ & $2.40 \mathrm{E}-01$ & $2.25 \mathrm{E}-01$ & $5.06 \mathrm{E}-01$ \\
\hline L-threonine & 0.43 & $3.89 \mathrm{E}-03$ & $7.72 \mathrm{E}-01$ & $3.54 \mathrm{E}-01$ & $4.02 \mathrm{E}-01$ \\
\hline adenosine & -3.67 & $7.00 \mathrm{E}-03$ & $6.61 \mathrm{E}-01$ & $8.21 \mathrm{E}-01$ & $2.84 \mathrm{E}-01$ \\
\hline glycine & 0.26 & $7.28 \mathrm{E}-03$ & $8.04 \mathrm{E}-01$ & $2.44 \mathrm{E}-01$ & $1.85 \mathrm{E}-01$ \\
\hline DL-isoleucine & 0.6 & $1.39 \mathrm{E}-02$ & $8.63 \mathrm{E}-02$ & $3.13 \mathrm{E}-01$ & $3.56 \mathrm{E}-01$ \\
\hline phosphoric acid & -0.35 & $1.80 \mathrm{E}-02$ & $9.14 \mathrm{E}-02$ & $2.32 \mathrm{E}-02$ & $9.13 \mathrm{E}-02$ \\
\hline L-proline & 0.50 & $2.00 \mathrm{E}-02$ & $6.02 \mathrm{E}-01$ & $7.89 \mathrm{E}-01$ & $1.73 \mathrm{E}-01$ \\
\hline
\end{tabular}

Table 1. Indole-3-propionic acid is significantly increased in the serum in IF vs AL.

Listed are metabolites differentially enriched in the serum of IF vs AL treated mice. Shown are the log fold change (logFC), IFvsAL p-value, vancomycin vs non-vancomycin (Ab vs non-Ab) p-value and the interaction $\mathrm{p}$ value between intermittent fasting and vancomycin treatment (interaction). R-square (Rsq, the coefficient of determination) showed the highest predictability (79.4\%) for indole-3-propionic acid.

\section{Figures}

a

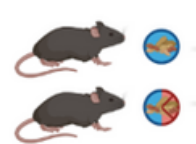

b

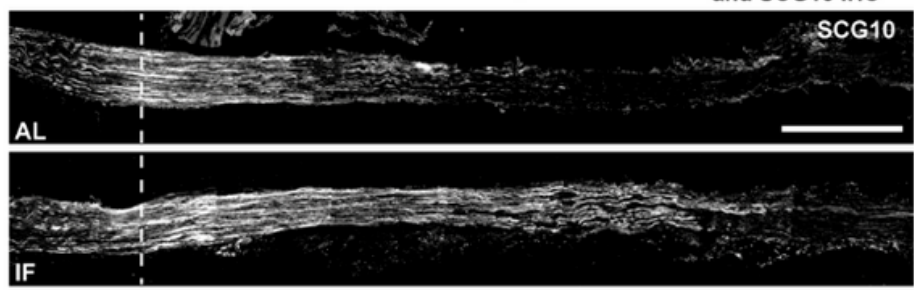

d

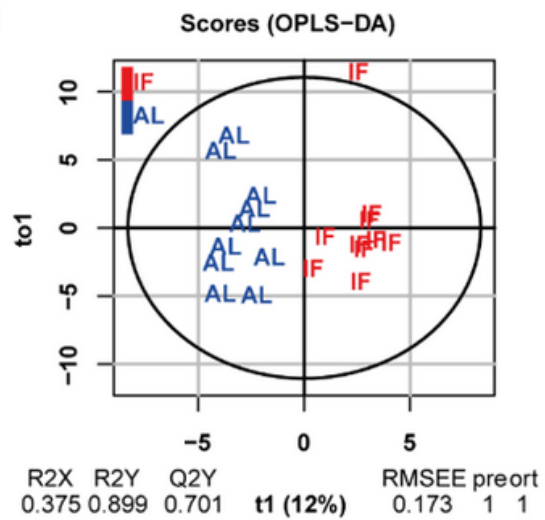

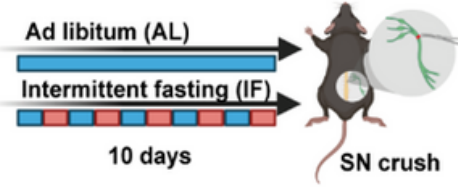

$\underset{72 \mathrm{~h}}{\longrightarrow}$ nerve dissection and SCG10 IHC

\begin{tabular}{|lcc|}
\hline Metabolite & logFC & p-value \\
3-indolelactic acid & 1.47 & $3.15 \mathrm{E}-06$ \\
2,3-butanediol & 1.37 & $5.34 \mathrm{E}-03$ \\
xylose & 1.11 & $1.17 \mathrm{E}-02$ \\
indole-3-propionic acid & 0.83 & $2.00 \mathrm{E}-03$ \\
D-glucose-6-phosphate & 0.8 & $4.89 \mathrm{E}-02$ \\
3-phosphoglyceric acid & 0.61 & $3.78 \mathrm{E}-02$ \\
DL-isoleucine & 0.60 & $2.86 \mathrm{E}-03$ \\
L-leucine & 0.57 & $1.63 \mathrm{E}-02$ \\
L-threonine & 0.43 & $5.10 \mathrm{E}-03$ \\
L-valine & 0.36 & $1.93 \mathrm{E}-02$ \\
glycine & 0.26 & $1.39 \mathrm{E}-02$ \\
phosphoric acid & -0.35 & $2.97 \mathrm{E}-02$ \\
adenosine & -3.67 & $3.93 \mathrm{E}-02$ \\
\hline
\end{tabular}

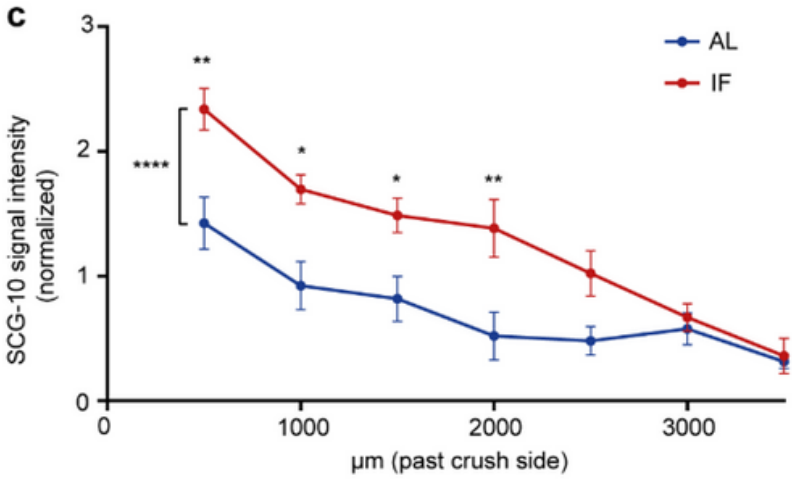

f Indole-3-propionic acid
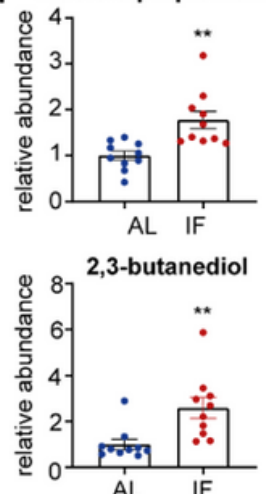

3-indolelactic acid
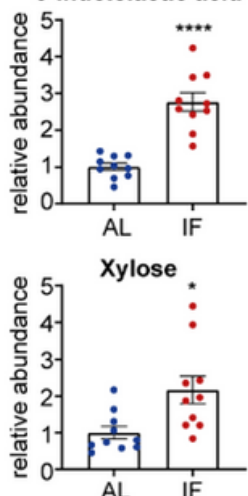

\section{Figure 1}

Intermittent fasting promotes axonal regeneration affecting gut microbiota metabolites a. Schematic of the experiment presented in b-d. b. Micrographs showing representative longitudinal sections of sciatic 
nerves $72 \mathrm{~h}$ after SNC immunostained with SCG-10 (Scale bar: $1000 \mu \mathrm{m})$. The dashed line indicates the crush site. c. Quantification of normalised SCG-10 intensity at the indicated distances ( $N=8$ nerves from 4 mice per group with a bilateral sciatic nerve crush; $* \star \star \star P<0.0001, * * P<0.01, * P<0.05$, two-way-ANOVA with Tukey post-hoc test, mean \pm SEM). $d$. Score plot of the OPLS-DA on GC-MS-based metabolic profiles ( $N=$ 10 per group). e. Table listing significantly upregulated and downregulated metabolites in IF vs AL serum $(P<0.05)$. Shown are the logarithmic fold changes $(\log F C)$ and the $p$-value between $I F$ and $A L$ groups ( $N=A L: 10, I F: 10$ mice per group). $f$. Bar graphs plotting the relative abundance of gut microbiome derived metabolites from the GC-MS analysis: indole-3-propionic acid, 3-indolelactic acid 2, 2.3-butanediol 2 and xylose 2 ( $N=$ AL: 10, IF:10; ${ }^{* \star} p<0.01,{ }^{* \star *} p<0.001,{ }^{\star \star * \star} p<0.0001$, mean $\left.\pm S E M\right)$.

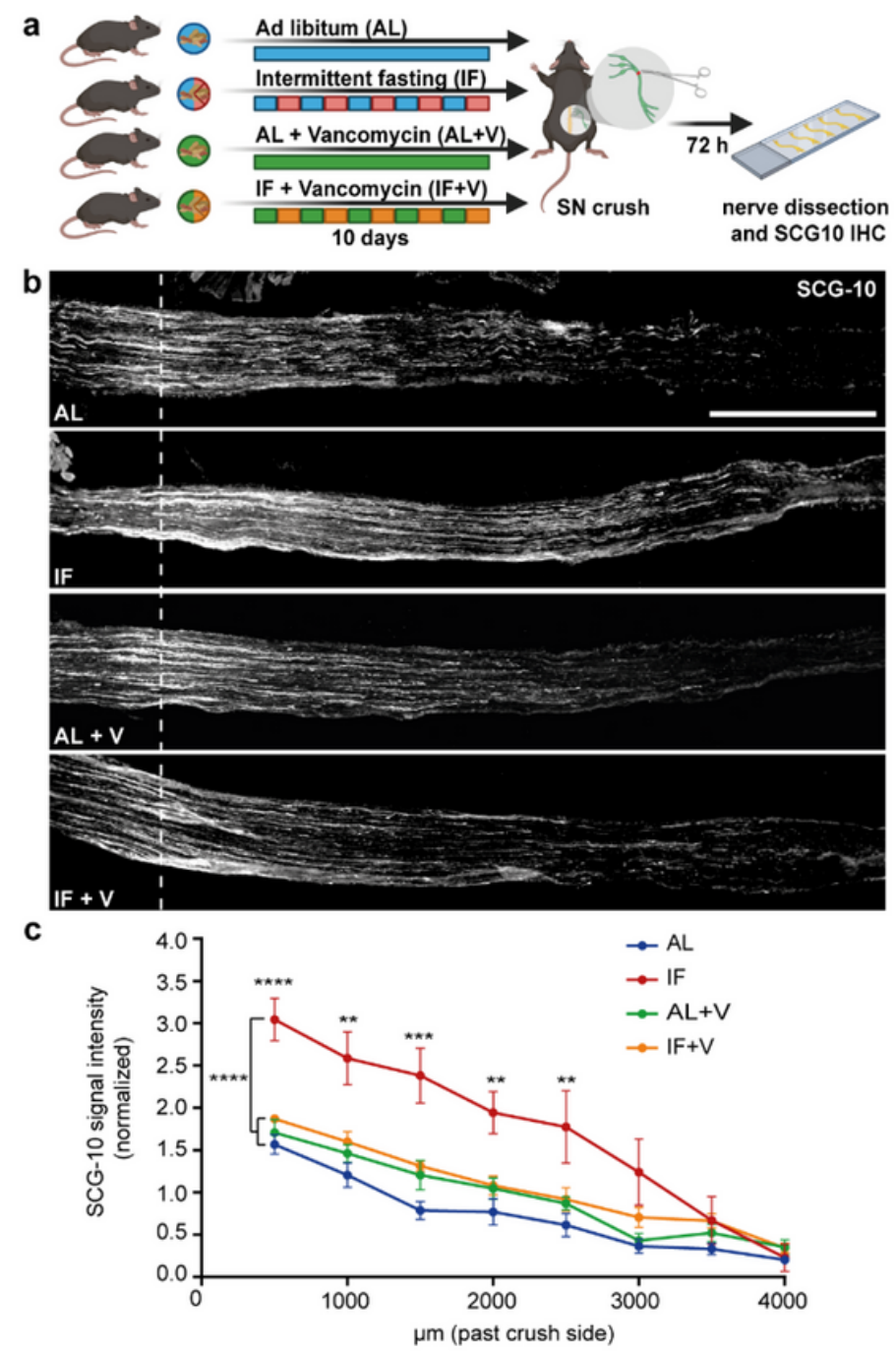

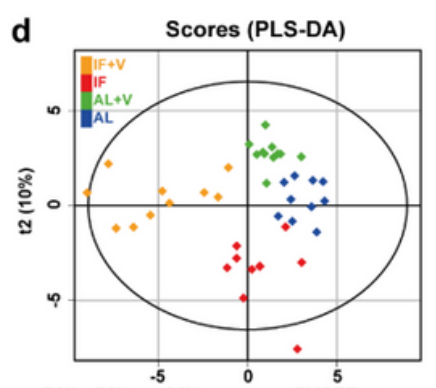

$\begin{array}{lllll}R 2 X & R 2 Y & \text { Q2Y } & \text { t1 (16\%) } & \text { RMSEEpre } \\ 0.261 & 0.525 & 0.374 & \text { t1 }\end{array}$
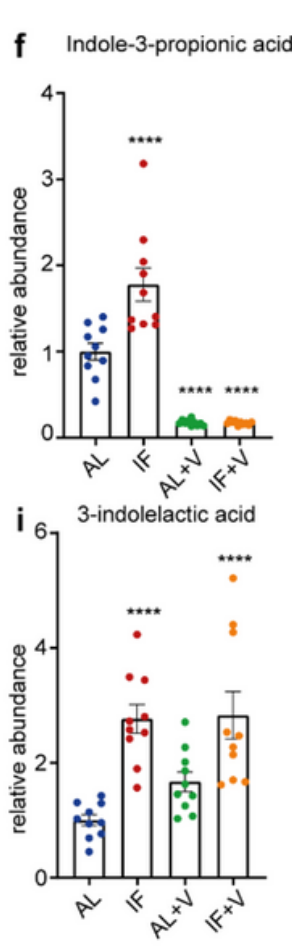
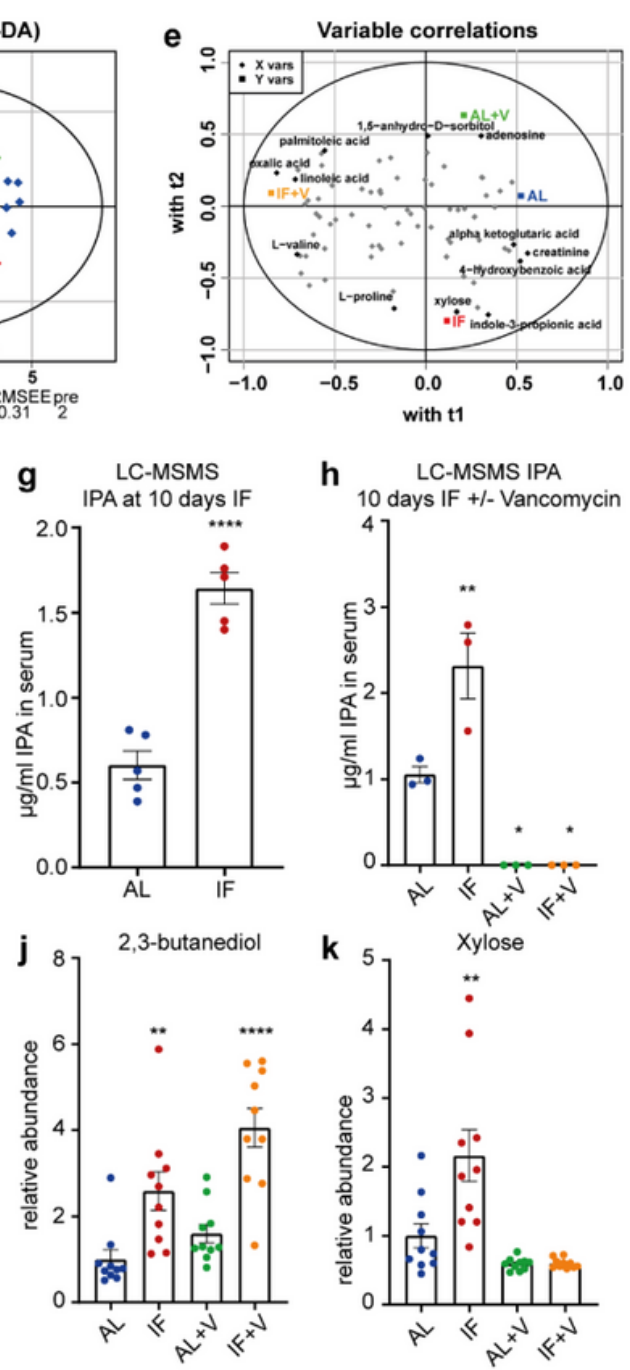

h $\begin{gathered}\text { LC-MSMS IPA } \\ 10 \text { days IF +/- Vancomycin }\end{gathered}$
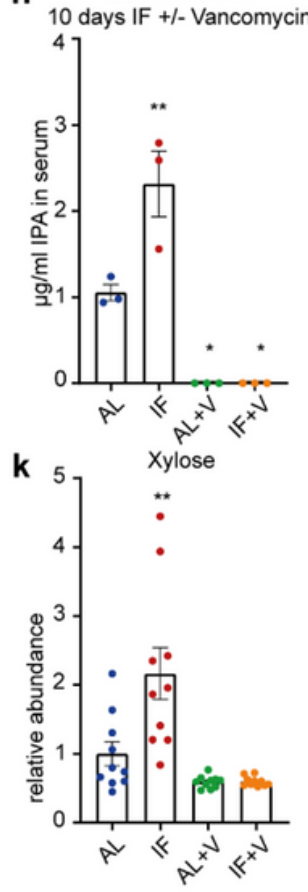

Figure 2

Intermittent fasting promotes axonal regeneration via a gut gram+ microbiome dependent mechanism that produces IPA a. Schematic of the experiment presented in b.-c. 10 days IF or AL +/-vancomycin and 3 days post sciatic nerve crush. b. Micrographs of representative longitudinal sections of sciatic nerves for all groups immunostained with SCG-10. Scale bar: $1000 \mu \mathrm{m}$. The dashed line indicates the crush site c. Quantification of normalised SCG-10 intensity at the indicated distances from the crush site ( $\mathrm{N}=6-7$ nerves 4 mice per group with a bilateral sciatic nerve crush; ${ }^{*} * \star P<0.0001,{ }^{*} \mathrm{P}<0.01$, two-way-ANOVA with 
Tukey test, mean \pm SEM) d-k. GC-MS metabolomics analysis was conducted from serum after 10 days of IF/AL with and without vancomycin co-treatment. d. Score plot of the PLS-DA analysis performed on normalized GC-MS metabolomic data $(\mathrm{N}=10)$. e. Variable Correlations analysis models the overlap between loadings and score plot showing metabolites that are primarily represented in specific groups. $f$. Indole-3-propionic acid is completely depleted by vancomycin treatment. (N= AL: 8, IF:9 mice per group; $\star \star \star * P<0.01$, one-way ANOVA, Dunnett's multiple comparisons test, $p$-value indicates comparison to $A L$, mean \pm SEM). g. LC-MS/MS measurements of IPA in serum from mice following 10 days of intermittent fasting vs. ad libitum ( $N=5$, Student's t-test, mean $\pm S E M)$ h. LC-MS/MS measurements of IPA in serum from mice following 10 days of IF vs $A L$ with and without vancomycin treatment ( $N=3$, one-way ANOVA, Dunnett's multiple comparisons test, $p$-value indicates comparison to $A L$, mean $\pm S E M) ~ i-k$. Relative abundance of 3-indolelactic acid 2, 2.3-butanediol 2 and xylose 2 across experimental groups ( $N=A L: 10$, IF:10 mice per group; ${ }^{* *} \mathrm{P}<0.01,{ }^{\star \star * *} \mathrm{P}<0.0001$, one-way ANOVA, Dunnett's multiple comparisons test, $\mathrm{p}$ value indicates comparison to $\mathrm{AL}$ control group, mean \pm SEM).

a
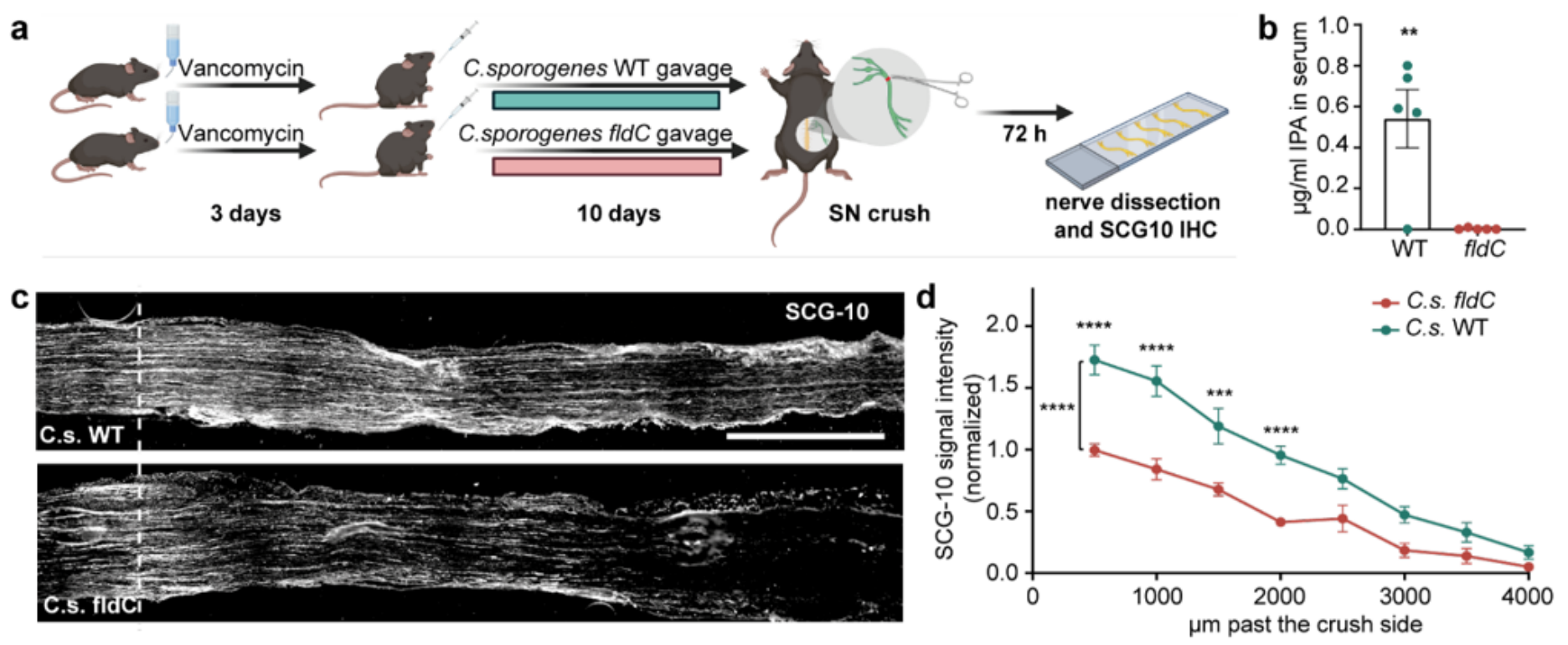

e
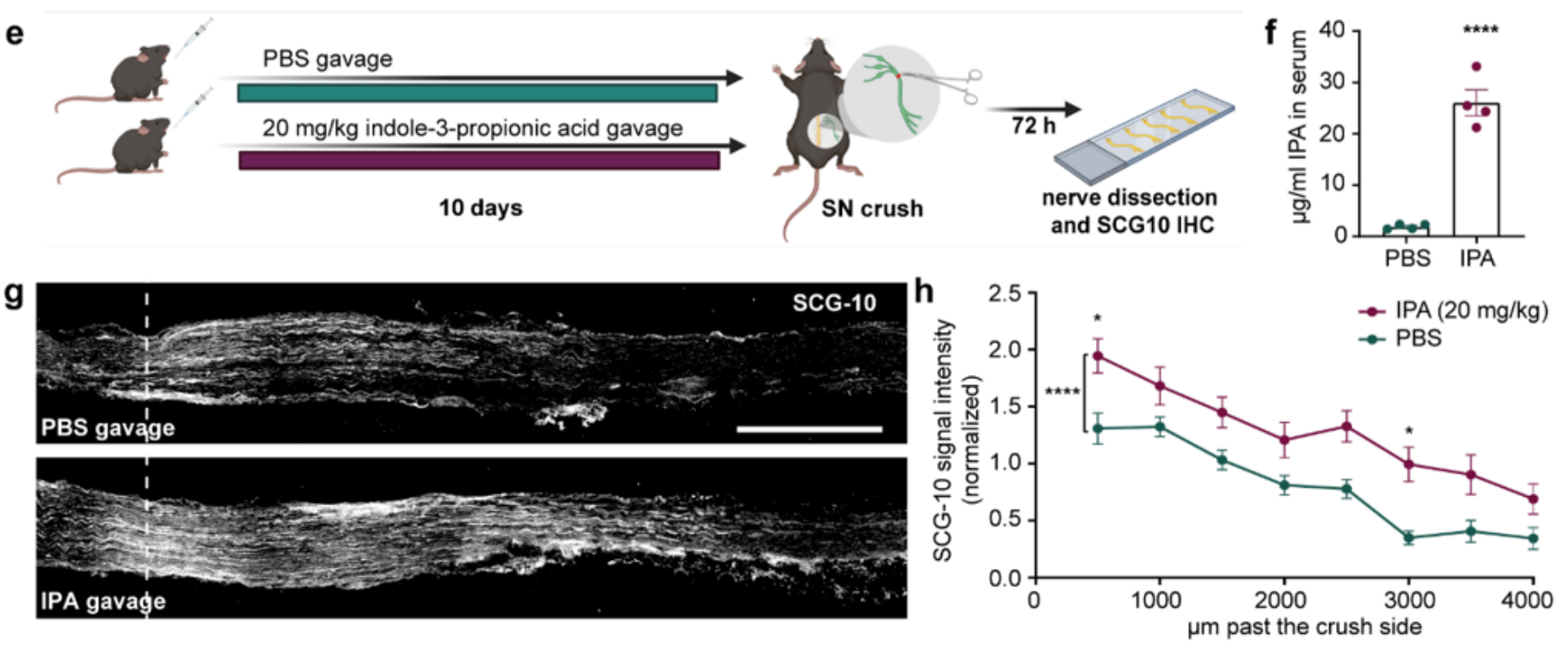


\section{Figure 3}

Indole-3-propionate promotes axonal regeneration of DRG neurons after SNC a. Schematic of the experimental design. b. LC-MS/MS measurements of IPA in serum from mice following 10 days of Clostridium sporogenes wildtype vs mutant (fldC) transplantation ( $\mathrm{N}=5$, Student's unpaired t-test, mean \pm SEM) c. Micrographs of representative longitudinal sections of sciatic nerves $72 \mathrm{~h}$ after SNC, immunostained with SCG-10. Scale bar: $1000 \mu \mathrm{m}$. The dashed line indicates the crush site. $d$.

Quantification of SCG-10 intensity at the indicated distances plotted as a function of the distance from the crush site ( $N=8$ nerves from 4 mice per group with a bilateral sciatic nerve crush; ${ }^{\star \star \star \star} p<0.0001$, $\star * \star \mathrm{p}<0.001$, two-way-ANOVA with Sidak post hoc test, mean \pm SEM). e. Schematic of the experimental design. f. LC-MS/MS quantification of IPA in serum from mice following 10 days of IPA gavage $(\mathrm{N}=4$, Student's unpaired t-test, mean \pm SEM). g. Micrographs of representative sections of sciatic nerves immunostained with SCG-10. Scale bar: $1000 \mu \mathrm{m}$. The dashed line indicates the crush site. $\mathrm{h}$. Quantification of normalised SCG-10 intensity at the indicated distances. ( $\mathrm{N}=8$ nerves from 4 mice per group with a bilateral sciatic nerve crush; ${ }^{* * \star *} \mathrm{p}<0.0001,{ }^{*} \mathrm{p}<0.05$, two-way-ANOVA with Sidak posthoc test, mean \pm SEM). 


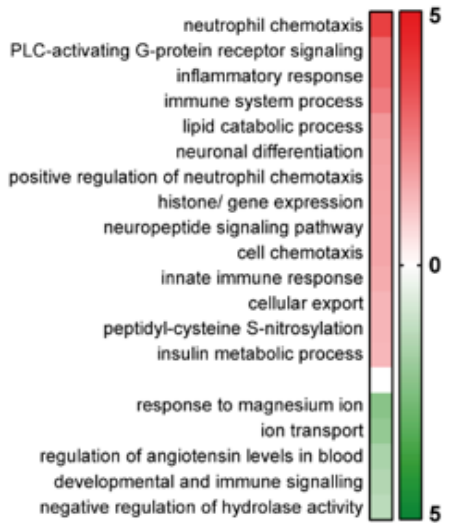

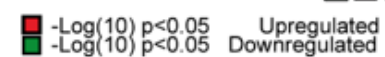

b

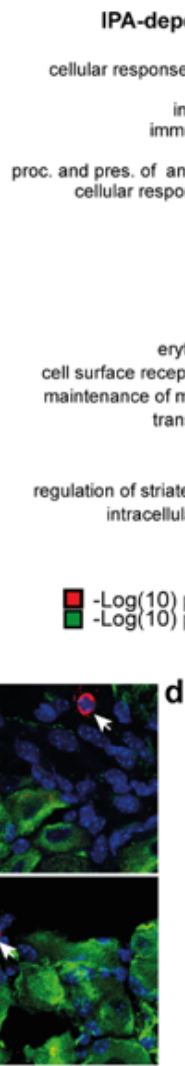

A-dependent BP-GO

ponse to interferon-gamma
cell adhesion inflammatory response inmune system response immune defense to interferon-beta cell-matrix adhesion cell proliferation NS development GPCR signalling
development

muscle contraction ansmembrane transport developmen
ion transport ion transport nal transduction spermatogenesis cation transpo

$\operatorname{og}(10) p<0.05 \quad \begin{array}{r}\text { Upregulated } \\ p<0.05\end{array}$ e
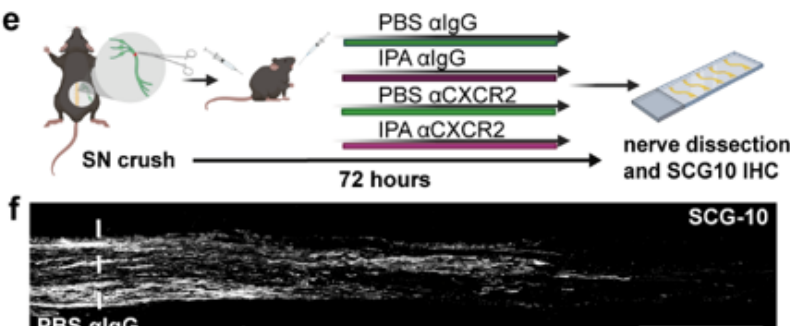

PBS glgG

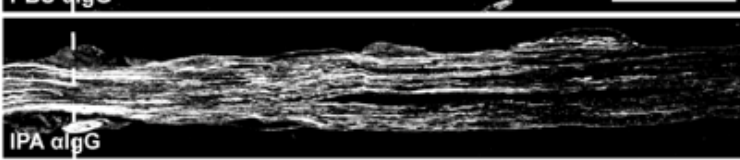

IPA aige

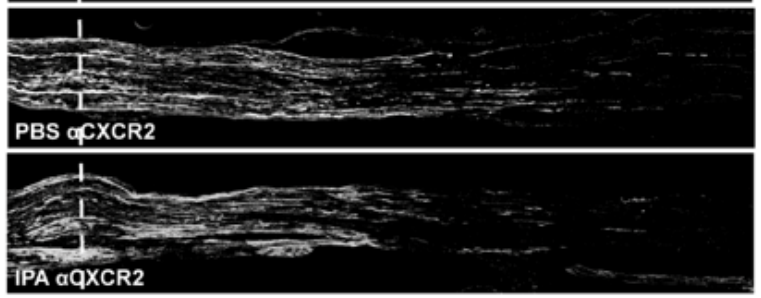

g
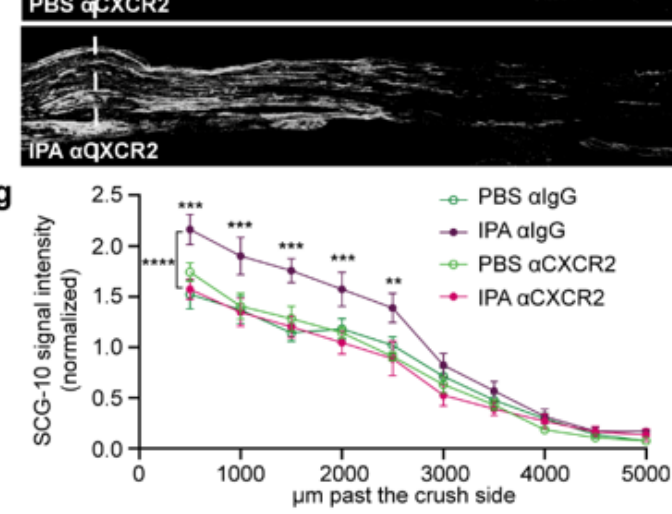

h
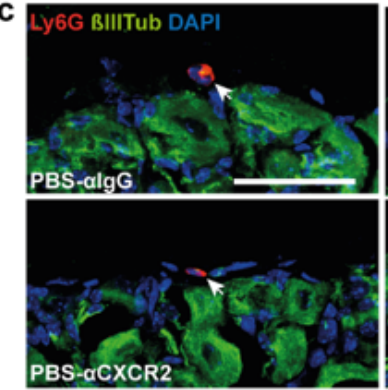

IPA-algC

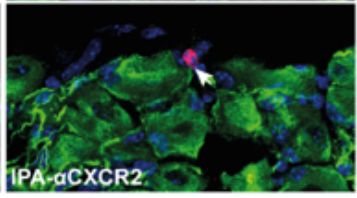

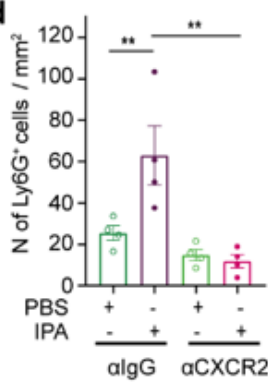
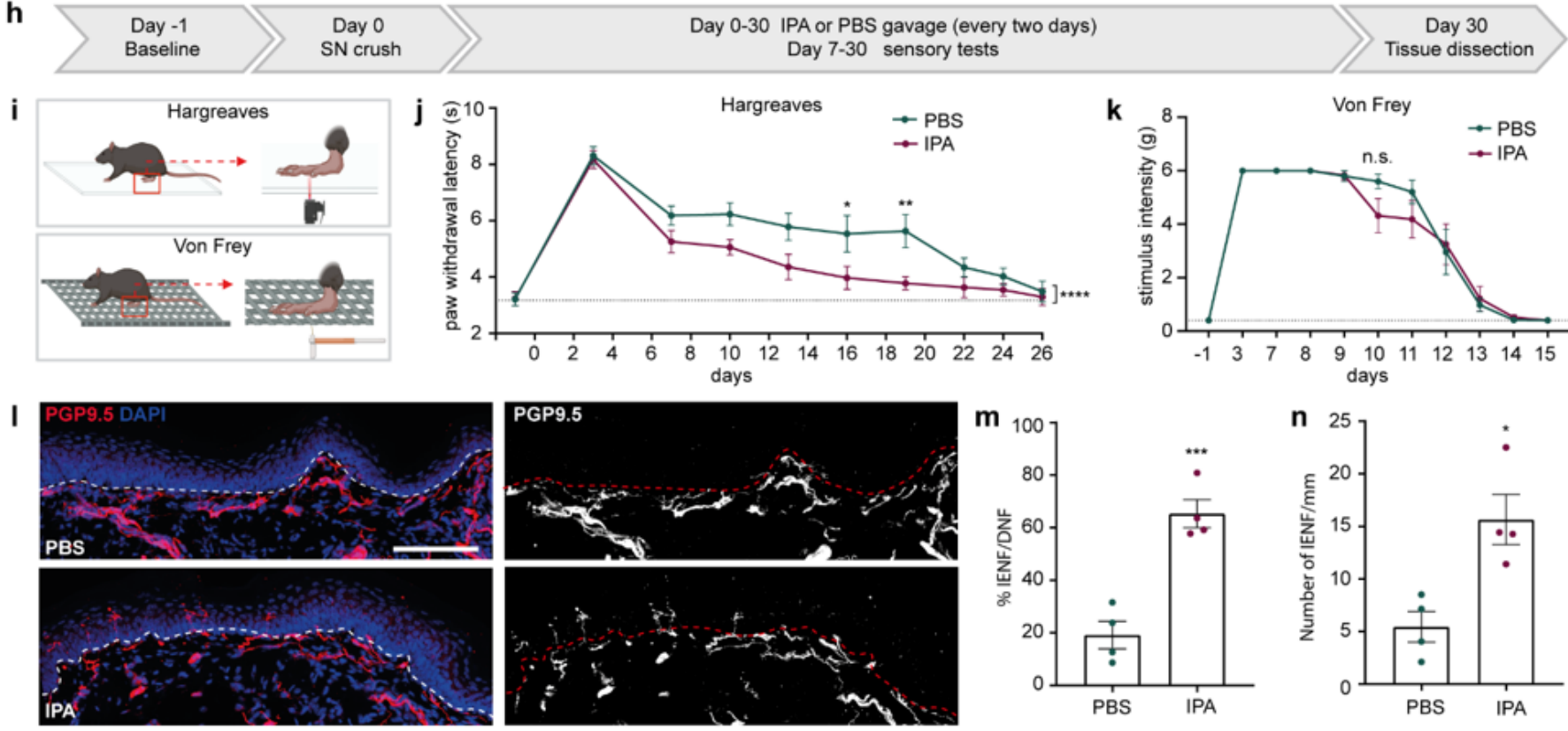

\section{Figure 4}

IPA-dependent axonal regeneration requires neutrophil chemotaxis and accelerates recovery of thermal heat sensation and epidermal innervation a-b. GO biological process analysis of IPA-dependent up- and downregulated genes $(|1.5|$

\section{Supplementary Files}


This is a list of supplementary files associated with this preprint. Click to download.

- Sl.docx 